OPEN ACCESS

Edited by: Manuel Perea,

Universitat de València, Spain

Reviewed by:

Judith F. Kroll,

Pennsylvania State University,

United States

Sarah Schimke,

Universität Münster, Germany

${ }^{*}$ Correspondence:

Hadas Shintel

hadas_s@mla.ac.ll

Specialty section: This article was submitted to

Language Sciences,

a section of the journal

Frontiers in Communication

Received: 12 December 2017 Accepted: 10 July 2018

Published: 31 July 2018

Citation:

Shintel $H$ and Faust $M(2018)$ Warming Up the Language Engines:

Short-Term Second Language Use Increases Subsequent Fluency.

Front. Commun. 3:32.

doi: 10.3389/fcomm.2018.00032

\section{Warming Up the Language Engines: Short-Term Second Language Use Increases Subsequent Fluency}

\author{
Hadas Shintel ${ }^{1,2 *}$ and Miriam Faust ${ }^{2,3}$ \\ ${ }^{1}$ Department of Psychology, The Center for Academic Studies, Or Yehuda, Israel, ${ }^{2}$ Gonda Multidisciplinary Brain Research \\ Center, Bar Ilan University, Ramat Gan, Israel, ${ }^{3}$ Department of Psychology, Bar Ilan University, Ramat Gan, Israel
}

Subjective experience suggests that using one's second language $(\mathrm{L} 2)$ becomes easier after an initial period of adjustment. We report the results of an experiment suggesting that even a brief period of L2 use can facilitate subsequent retrieval. Native Hebrew speakers completed letter and category verbal fluency tasks in English. In a subsequent experimental session, participants performed a short comprehension or production task, either in Hebrew (L1) or in English (L2), and then completed an additional round of the English fluency tasks. English use resulted in a reliable improvement in letter fluency, associated with executive functioning. However, there was no reliable improvement in category fluency, associated with lexical knowledge. No reliable improvement in the fluency tasks was observed following Hebrew use. Results suggest that even a brief period of $L 2$ use can facilitate retrieval, by increasing the relative activation of $L 2$. Furthermore, improvement did not result from priming specific lexical items, suggesting increased relative activation affects the L2 lexicon as a whole.

Keywords: second language, verbal fluency, executive function, word retrieval, non-native speakers

\section{INTRODUCTION}

Individuals who need to use their second language (L2) often report feeling that L2 use becomes less effortful and more fluent after an initial period of adjustment. The subjective feeling of enhanced fluency can arise even after a relatively short period of L2 use (e.g., at the end of a conversation relative to its beginning), and is transient, suggesting it cannot be attributed to increased proficiency. A colleague, a native speaker of Hebrew living in the USA, termed this experience "the Monday-Friday effect," referring to the subjective ease of using English on Friday, after a week of using predominantly English, relative to Monday, after a weekend of using predominantly Hebrew. However, it is unclear whether this subjective experience is associated with an objective increase in L2 fluency, defined as the ability to "mobilize one's linguistic resources in the service of real time communication, i.e. to produce (and comprehend) speech at relatively normal rates" (Skehan, 1996, p. 48).

One way in which L2 use can boost subsequent fluency is by increasing the relative activation of L2. Models of bilingual language processing suggest that different languages differ in their activation level within the bilingual's language system. This activation level, however, is neither fixed nor dependent solely on the individual's level of proficiency, but may vary depending on context and task demands (BIA model, Dijkstra and Van Heuven, 1998; cf. The Inhibitory Control model, Green, 1998; the language mode hypothesis, Grosjean, 2001). An increase in the relative activation of L2 may stem from inhibitory mechanisms operating to reduce the activation level of 
the dominant first language (L1). For example, studies found evidence for lesser L1 performance immediately following L2 use or in an L2 context, possibly resulting from inhibition of L1 during L2 use (Meuter and Allport, 1999; Levy et al., 2007; Linck et al., 2009; see Kroll et al., 2008 for a review of the role of inhibition in bilingual production. For an opposing view regarding the role of inhibitory processes in highly proficient balanced bilinguals see Costa and Santesteban, 2004). Inhibition of L1 shifts the relative activation of the two languages and thus may enhance the accessibility of L2 representations. Alternatively, an increase in the relative activation of L2 may be driven by selection mechanisms operating to preferentially increase the activation level of L2 or lower the threshold for L2 selection (Poulisse and Bongaerts, 1994; Grosjean, 2001).

Most previous studies have examined language control using a language switching paradigm in which participants are asked to name words in L1 or in L2, based on a predetermined cue (e.g., Meuter and Allport, 1999; Costa and Santesteban, 2004). Meuter and Allport (1999) found a greater switch cost (in terms of naming latency) involved in switching to L1 than to the weaker L2. The asymmetrical switch cost can be explained by assuming that L2 naming entails a stronger active inhibition of the dominant $\mathrm{L} 1$ than vice versa. This suggests that L2 use confers a temporary benefit by increasing the relative activation of L2. However, the typical context of language use does not involve constant language switching based on an arbitrary cue. Given the importance of task demands and the experimental context to the interplay between bilinguals' two languages (Grosjean, 2001; Wu and Thierry, 2010), our aim here was to examine whether the benefit provided by L2 use goes beyond a fleeting effect on a subsequent trial, and reflects a more gradual adjustment of the relative activation of both languages. Furthermore, most studies examined the effect of L2 use on the accessibility of L1 words (and the resulting L1 interference). Here we wanted to examine whether changes in the relative activation of the two languages can actually increase L2 performance. A brief period of L2 use may temporarily enhance the accessibility of L2 representations, thereby making cognitive resources, typically needed to access L2, available for other aspects of language production and consequently enhance L2 fluency. The construct of fluency is a multidimensional construct, which consists of several facets (see Tavakoli and Skehan, 2005) such as speed fluency (e.g., speech rate), breakdown fluency (e.g., number of filled and unfilled pauses), and repair fluency (e.g., false starts). Here we focus on effective lexical retrieval, as one essential process underlying fluency. Fluency can be viewed as a component of proficiency; however the two are distinct in several ways. First, proficiency depends on one's linguistic knowledge (e.g., lexical knowledge). Second, our focus here is on fluency as a fluid and transient measure that can very across contexts, whereas proficiency is typically viewed as a more stable ability.

A related issue is whether the processes involved in language selection and control operate on a local or a global level. A local control process will pertain only to specific L1 items such as translation equivalents. For example Levy et al. (2007) found that retrieving words in Spanish reduced the accessibility of the corresponding words in English. Alternatively, control processes may operate globally, pertaining to the lexicon as a whole (cf. Meuter, 2005 for a discussion). In this case, inhibition may be evident even in a context that does not require constant language switching (see Guo et al., 2011; Misra et al., 2012).

The present study was aimed at investigating whether there is a benefit to L2 fluency, in particular L2 lexical retrieval, from a relatively brief period of L2 use. For this purpose, we examined the effect of a short-term use of English by native Hebrew speakers on subsequent English verbal fluency. We used two verbal fluency measures, letter fluency, in which participants are asked to generate as many items as possible beginning with a specific letter, and category fluency, in which participants are asked to generate as many exemplars as possible from a specific semantic category. While both measures involve lexical retrieval, they tap into different underlying cognitive functions (cf. Bialystok et al., 2009) and engage different brain mechanisms (see Luo et al., 2010). In the letter fluency measure, the search is guided by the first phoneme. Because such phonology-based search is less practiced and involves more effortful monitoring, it places greater demands on executive control (Luo et al., 2010). Number of words generated in this task is more correlated with switching between phonemic categories than with producing phonemically-related clusters (Troyer et al., 1997). As a result, performance in this task is associated with executive control (Bialystok et al., 2008) and is mediated by frontal regions (Baldo et al., 2006). On the other hand, phonological categories are typically broad and contain many high-frequency words that even nonnative speakers are expected to know. Note that successful performance in the letter fluency requires access to English phonology (moreover, the languages included in the study utilize distinct scripts) and cannot be systematically mediated by Hebrew (only insofar as translation equivalents happen to begin with the first phoneme). The letter fluency task does not require language switching. However, an increase in the relative activation of English may reduce the load on executive processes on a subsequent letter fluency task, resulting in better lexical retrieval. If the executive burden entailed by L2 use is reduced following initial adjustment, we would expect an increase in English word fluency in a task that engages executive control.

In the category fluency measure, participants need to search through semantic memory. High scores in this task necessitate the retrieval of low-frequency exemplars; thus it heavily relies on vocabulary and semantic memory. Indeed previous research has found performance in this task is correlated with vocabulary size (Luo et al., 2010) and is mediated by temporal regions (Baldo et al., 2006). Previous research found a bilingual disadvantage on category fluency, possibly due to weaker connections between semantic and phonological representations (Gollan et al., 2002) and smaller vocabulary relative to monolinguals (Portocarrero et al., 2007; see also Bialystok et al., 2008, experiment 2, for evidence suggesting the bilingual disadvantage disappears when controlling for vocabulary size). On the other hand, given the tight connections between items within a semantic category, it places lower demands on executive control. Indeed performance in this task did not correlate with executive control 
(Luo et al., 2010). Moreover this task shows greater sequential priming between items then the letter task (Schwartz et al., 2003) suggesting it is more susceptible to the influence of local spreading activation. Lastly, in contrast to the letter task, performance in the category task can be systematically mediated through Hebrew, by relying on L1-L2 word associations. Because category fluency is constrained by relatively stable lexical proficiency, initial adjustment to L2 may not increase fluency.

Finally, we examined whether the mode of L2 use (comprehension vs. production) will differentially affect subsequent L2 fluency. The idea that language control involves inhibition of the non-target language has been incorporated in models of comprehension (Dijkstra and Van Heuven, 1998) as well as production (Green, 1998). Both L2 comprehension and L2 production may increase the relative activation of L2, and therefore increase L2 fluency. However, in contrast to bilingual language production studies (e.g., Meuter and Allport, 1999), previous studies of bilingual language comprehension did not find asymmetrical switch costs (e.g., Thomas and Allport, 2000). If the larger switch cost to L1 than to L2 indeed reflects the linhibition of L1 during L2 production, the lack of asymmetrical switch costs in comprehension can be interpreted as suggesting a smaller involvement of inhibitory processes (for further evidence suggesting greater involvement of inhibitory processes in language production using a different paradigm, see Declerck and Philipp, 2018). Furthermore, given that the fluency tasks require word production, it may be that actual L2 production, that involves practicing retrieval of the word's phonological form, is essential for increased fluency.

\section{MATERIALS AND METHODS}

\section{Participants}

The experiment was approved by the ethics committee of the psychology department at Bar Ilan University. One Hundren and Seventy Two Bar Ilan University students participated in the study. All participants were native Hebrew speakers, born in Israel, who learned English as a second language and did not use it at home (mean age of beginning to learn English $=8.7$, see Table 1). To ensure basic proficiency in English and minimize variability across participants, all participants had a score of 100 points and above, out of 150, in the English section of the university entrance psychometric exam. The grade on the entrance exam is used by the university to determine the relevant level of English courses students will be assigned to. A grade of 100 implies participants were classified at English level 5 ("Low Advanced") or above, out of 7 language levels, ranging from "Intensive Beginners" to "Exempt." To reduce the effect of outlier cases, four participants whose scores on the letter fluency pretest were 2.5 standard deviations or more above ( 2 participants, mean fluency $=23.5$, compared to a total mean of 12.12) or below the total mean ( 2 participants, mean fluency $=3$ ) were excluded from further analysis (this did not affect the overall pattern of results). Data from eight additional participants were not analyzed due to experimenter's error or equipment failure (6 participants), cell phone ringing during the fluency task (1 participant), or not following instructions (1 participant).
TABLE 1 | Participant characteristics in the different conditions.

\begin{tabular}{lcccc}
\hline Condition & $\begin{array}{c}\text { English } \\
\text { level }\end{array}$ & $\begin{array}{c}\text { Age of } \\
\text { acquisition }\end{array}$ & $\begin{array}{c}\text { English } \\
\text { proficiency }\end{array}$ & $\begin{array}{c}\text { English } \\
\text { use }\end{array}$ \\
\hline $\begin{array}{l}\text { English production } \\
\begin{array}{l}\text { English } \\
\text { comprehension }\end{array}\end{array}$ & $6.23(0.77)$ & $8.6(1.87)$ & $5.06(1.02)$ & $3.9(0.89)$ \\
$\begin{array}{l}\text { Hebrew } \\
\text { production }\end{array}$ & $6.38(0.75)$ & $8.77(1.27)$ & $5.13(0.78)$ & $3.89(1.02)$ \\
$\begin{array}{l}\text { Hebrew } \\
\text { comprehension }\end{array}$ & $6.23(0.76)$ & $8.86(1.53)$ & $5.24(0.96)$ & $3.88(1.26)$ \\
\hline
\end{tabular}

Standard deviations are shown in parentheses.

\section{Design and Procedure}

Participants completed two rounds (pretest and posttest) of the verbal fluency tasks in English; the difference in performance served as the dependent measure. Pretest and posttest were identical across conditions. The experimental manipulation consisted of varying the language context preceding the fluency posttest (Figure 1). Language context indicates the language used in the interim task preceding the fluency posttest; all fluency tasks themselves were performed exclusively in English. Language context (English vs. Hebrew) and mode of use (Comprehension vs. Production) were manipulated between participants, resulting in four experimental conditions. Participants were randomly assigned to conditions, 32 in each group. Participants completed the experiment in two sessions. The second session was conducted 3-8 days after the first session (mean $=5.37$, no significant difference between conditions, all $F$ s $<1$ ). The first session was identical for all participants and included two verbal fluency tasks in English (i.e., fluency pretest), one letter fluency task and one category fluency task (to reduce the effects of practice, participants performed each task only once per session). In the letter fluency task participants were given $60 \mathrm{~s}$ to generate as many words as possible, beginning with the letter $F$ or $S$ ("I want you to tell me as many words as you can think of that begin with that letter."), excluding proper names and derivatives. In the category fluency task, participants were given $60 \mathrm{~s}$ to generate as many exemplars belonging to the semantic categories of animals or fruits ("I want you to tell me the names of all the things that you can think of that belong to that category.") The first few minutes of the session were conducted in Hebrew; the experimenter greeted the participants, had them sign Hebrew consent forms and explained that the session includes language tasks in English. After that stage, to minimize Hebrew use, instructions for both fluency tasks were presented in written English and spoken communication between participants and the experimenter was kept to a minimum.

In the second session participants first completed a language task that required them to use either Hebrew (i.e., Hebrew context) or English (i.e., English context). Within each language context condition, half of the participants completed a production task, while the other half completed a comprehension task. The production task was a picture description task in which participants were asked to describe, as specifically as possible, 3 pictures and one picture story (e.g., a 


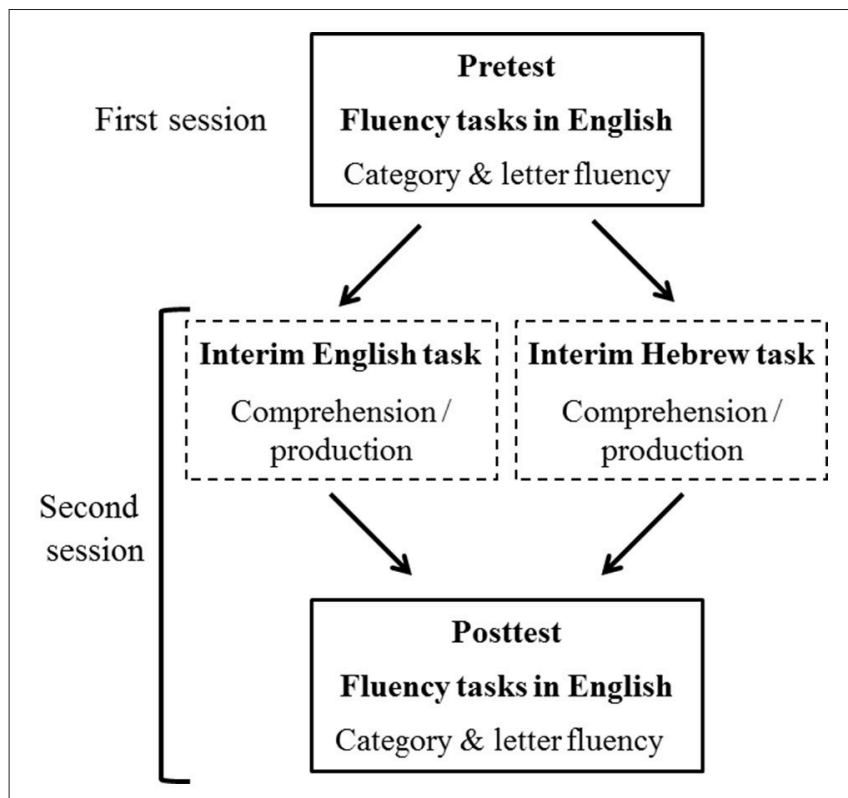

FIGURE 1 | |llustration of the experimental design.

train station scene), either in L1 or in L2. In the comprehension task, participants read written descriptions of the same pictures presented in the production task. To ensure that participants read the descriptions, the task included two rounds of a picture verification task in which participants indicated whether a presented picture (e.g., the described train station picture or one depicting a different train station scene) matches the described picture. Following these tasks, all participants completed another round of the English verbal fluency tasks with the complementary letter and category (fluency posttest). Order of fluency tasks was counterbalanced across participants within each experimental group, such that participants did not repeat the task with the same letter and category (e.g., half the participants completed the letter fluency task with the letter $F$ on the first session and $S$ on the second, and half the participants completed the task with $S$ on the first session and $F$ on the second). As in the first session, communication during the first few minutes of the session took place in Hebrew. Afterwards, to maintain the relevant language context for that condition, written instructions were presented in the language in which the task was to be performed, and spoken communication between participants and the experimenter was kept to a minimum. Instructions for the English verbal fluency tasks were written in English for all participants (same as in session 1). At the end of the second session, participants filled out a self-report questionnaire regarding English age of acquisition, proficiency (speaking, reading, writing, listening, and vocabulary), and degree of everyday use (speaking, listening, and reading). All questions were rated on a 7-point likert scale, with higher ratings indicating a greater degree of proficiency (1- very low, 7- excellent) and use (1- never, 7- on a daily basis).

The Hebrew conditions in which participants performed the interim language tasks in Hebrew served as a control condition that allowed us to estimate the effect of previous practice with the fluency tasks. Given that participants predominantly used Hebrew in everyday life, we expected L2 fluency in the Hebrew conditions to reflect the typical pattern of relative activation of Hebrew and English; we did not expect the short interim Hebrew tasks to have a further effect on L2 fluency. However, to further evaluate whether L2 fluency was affected by the immediately preceding Hebrew task, 32 additional participants participated in a non-linguistic control condition. English fluency pretest and posttest was identical to the experimental groups. However, in the second session, instead of a language task, participants completed a non-linguistic spatial pattern matching task in which they had to locate a specific pattern of Xs within a larger pattern. Performance in this condition provided a baseline against which performance in the Hebrew conditions can be compared. If using Hebrew immediately prior to the English fluency tasks results in reduced English verbal fluency, this should be reflected in a smaller fluency improvement in the Hebrew conditions, relative to the non-linguistic condition.

\section{Analysis}

Answers in the fluency tasks were recorded onto digital audiotape and analyzed. The score for each fluency task was the number of unique items generated in $1 \mathrm{~min}$, excluding errors and repetitions. Baseline performance in the pretest (across all participants) did not differ reliably between the $S$ and $F$ versions of the letter fluency task [mean $F=11.82(\mathrm{SD}=3.27)$, mean $S=12.49$ (3.41), $\left.t_{(126)}-1.15 p>0.25\right]$. However, in line with previous results (Gollan et al., 2002), baseline performance in the pretest differed between the animals and the fruits versions of the category task [mean animals $=12.73(4.6)$, fruit $=8.77$ (3.45), $\left.t_{(126)}=5.52, p<0.0001\right]$. To compare performance across different versions of the task, results were converted into $z$ scores. We calculated $z$ scores for both the pretest and posttests scores for each participant, relative to the mean and standard deviation of the pretest scores (calculated for each task version separately, collapsed across language context and mode of use conditions). Results from both fluency tasks were entered into two separate multiple regression analyses with posttest fluency as the dependent measure, and pretest fluency and the dummy-coded variables of context language and mode of use as predictors.

Given that some of the words used in the English comprehension and production tasks began with $S$ or $F$, improvement in letter fluency could result from priming these specific lexical items. To examine this possibility, we examined the descriptions presented in the English comprehension task and identified 42 words beginning with either $S$ or $F$. We then calculated the frequency of mention of these specific words in the pre- and post-test letter fluency tasks in the Hebrew and the English comprehension conditions. We focused on the comprehension conditions for this analysis because the comprehension task involved consistent presentation of the same words across participants, whereas in the production task the specific words used varied across participants. If improvement in letter fluency is due to repetition priming, there should be greater posttest increase in generating these items for participants 
in the English comprehension condition, actually presented with the words, relative to participants in the Hebrew comprehension condition, not presented with the words.

\section{RESULTS}

A MANOVA conducted on participants' English age of acquisition, language level, total proficiency, and total use revealed no significant differences between groups (see Table 1), all $F$ s $<1$. Only a minority of the participants $(n=20)$ had any proficiency at another language (L3). These were almost equally distributed across language context conditions (Hebrew: $n=11$, English: $n=9$ ).

Results on each fluency task (see Table 2 for untransformed means) were entered into a multiple regression analysis to determine if fluency pretest, language context, and mode of use significantly predicted fluency posttest performance ${ }^{1}$. For the letter fluency task, results (see Figure 2) indicated that the predictors explained $35 \%$ of the posttest variance $\left(R^{2}=0.352\right.$, $F=22.43, p<0.0001)$. Pretest scores significantly predicted improvement $\left[\mathrm{B}=0.66, t_{(124)}=8.06, p<0.0001\right]$. Critically, language context made a unique contribution to the prediction of improvement beyond that of the pretest scores $[B=0.36$, $t_{(124)}=2.24, p=0.027$ ], such that improvement was greater in the English language context condition (see Figure 2). Mode of use (comprehension vs. production) did not significantly predict improvement $(t<1)$.

A markedly different pattern emerged for the category fluency task. The predictors explained $19 \%$ of the posttest variance $\left(R^{2}=0.192, F=9.8, p<0.0001\right)$. Pretest scores significantly predicted improvement $\left[\beta=0.44, t_{(124)}=5.4, p<0.0001\right]$. Neither language context nor mode of use significantly predicted performance ( $t$ s $<1$, see Figure 3 ).

There was no significant fluency improvement on both tasks in the non-linguistic spatial control group (see Table 2, all $t \mathrm{~s}<1$, $p s>0.3, n s)$. In fact, the slight increase in letter fluency was directly comparable to the Hebrew condition, suggesting that the use of Hebrew in language tasks immediately prior to

\footnotetext{
${ }^{1}$ For both fluency tasks, adding a language context by mode of use interaction term did not significantly add to the model (letter fluency: $\mathrm{R}_{\text {full }}^{2}=0.356$, $\mathrm{R}_{\text {reduced }}^{2}=0.356$; category fluency $\mathrm{R}_{\text {full }}^{2}=0.192, \mathrm{R}_{\text {reduced }}^{2}=0.192$, all $F s<1, \mathrm{n} . \mathrm{s}$ ). The interaction was thus dropped from further analysis.
}

the English fluency task did not further reduce fluency. This result makes intuitive sense: given that Hebrew is participants' native and dominant language, and that they were immersed in a Hebrew context, they should be in a Hebrew language mode (Grosjean, 2001) whether they perform a language task in Hebrew or merely a spatial task that does not require linguistic mediation.

There are several reasons for these results. First, given that semantic categories are not as broad as letter categories and contain fewer words that L2 speakers would be expected to know, performance on this task is highly constrained by participants' vocabulary. As can be seen from the pretest baseline performance, participants were far more disadvantaged at category fluency then at letter fluency, relative to native English speakers (mean category $=10.75$, letter $=12.12$; compare to English monolinguals on the same categories/letters, reported by Gollan et al., 2002: mean category $=18.25$, letter $=15.1$ ). Indeed, category fluency performance typically shows a bilingual disadvantage (Gollan et al., 2002; Portocarrero et al., 2007), possibly due to bilinguals' smaller vocabulary (Bialystok et al., 2008). Although cross-language competition might have affected performance, competition alone is unlikely to damage performance to that degree on this particular task: as participants had a full minute and were able to selfcorrect, a delay caused by the activation of competing L1 items should not have been so detrimental. Cross-language intrusions occurred rarely for words unrelated to the English word (14 errors overall, 7 at each session, average per participant: 0.05). This suggests that participants' greater disadvantage on the category task does not reflect solely cross-language competition. Consistent with this, Gollan et al. (2002) found that bilinguals did not produce more errors than monolinguals (and almost no cross-language errors), despite their performance disadvantage. Furthermore, Gollan et al. found that allowing words from both languages did not improve category fluency, suggesting bilinguals' disadvantage did not stem solely from the activation of competing L1 items. If category fluency relies on lexical proficiency, adjustment to L2 that does not enhance proficiency would not result in improvement. Interestingly, the above studies found a bilingual disadvantage in category fluency, even though testing took place in an L2-dominant context, suggesting it persisted despite recent L2 use and task setting. Thus, the lack of improvement in category

TABLE 2 | Verbal fluency pretest and posttest (untransformed scores).

\begin{tabular}{|c|c|c|c|c|c|c|}
\hline Condition & \multicolumn{2}{|c|}{ Letter fluency } & Difference & \multicolumn{2}{|c|}{ Category fluency } & Difference \\
\hline English comprehension & $11.38(3.49)$ & $13.78(4.92)$ & 2.4 & $11.71(5.21)$ & $11.84(4.54)$ & 0.13 \\
\hline Hebrew production & $12.66(3.22)$ & $13.67(2.93)$ & 1.01 & $10.59(4.56)$ & $11.65(5.11)$ & 1.06 \\
\hline Hebrew comprehension & $12.59(3.31)$ & $12.84(3.53)$ & 0.25 & $10.13(3.98)$ & $11.38(5.38)$ & 1.25 \\
\hline
\end{tabular}

Standard deviations are shown in parentheses. 


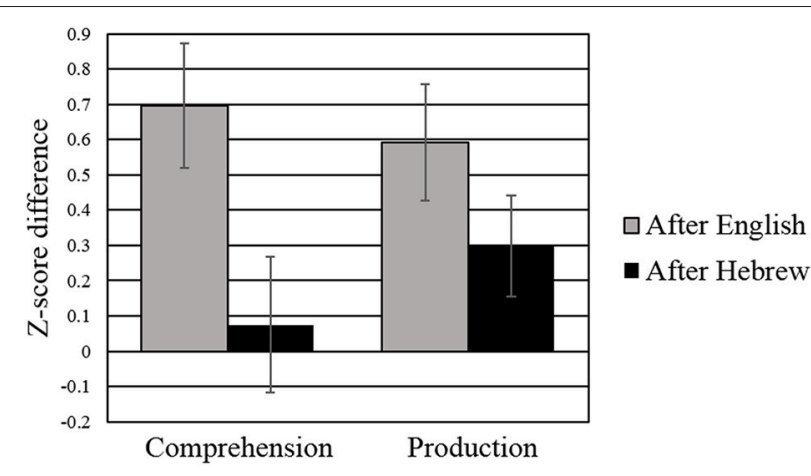

FIGURE 2 | Pre and post $z$-scores in the letter fluency task (error bars represent standard error).

fluency is not due to its semantic nature, but rather to its heavy reliance on vocabulary knowledge less available to L2 learners.

Second, category fluency can be systematically mediated through Hebrew by relying in Hebrew-English word associations. Indeed, informal comments by participants (e.g., "I am trying to come up with examples in Hebrew" or "I am blacking out even in Hebrew") suggest they tried to come up with relevant exemplars in Hebrew and then translate them into English. If participants use a translation strategy to perform the category task, inhibition of Hebrew following English use may not facilitate performance.

To gauge whether improvement in letter fluency following the intermediary English tasks resulted from priming specific lexical items that were used in these tasks (henceforth "presented words"), we analyzed the frequency of generating presented words during letter fluency pretest relative to posttest, in the English and the Hebrew comprehension conditions. If the processes underlying language selection operate on a local level, we would expect participants to retrieve more presented words after performing the comprehension task in English compared to Hebrew. In contrast, no difference between the conditions in the frequency of producing presented words would suggest that the increase in fluency does not reflect the increased activation of recently used items, and hence that the processes underlying language selection operate on a global level. In the English comprehension condition, mean frequency of generating presented words during pretest was 1.16, compared to 1.47 during posttest, an increase of 0.31 ( $p>$ 0.35 , n.s). As can be seen, even though participants have just read words beginning with $S$ or $F$, they did not exploit these in performing the fluency task. Moreover, a similar posttest increase was found in the Hebrew comprehension condition (mean pretest $=0.81$ posttest $=1.09$, no significant difference from English, $t<1$ ). Thus, even the minor posttest increase in fluency cannot be attributed to exposure to the words in the English condition and a resulting increase in their activation. This analysis suggests that the processes underlying enhanced L2 fluency operate on a global level, rather than via local item-specific processes that increase the activation of L2 words.

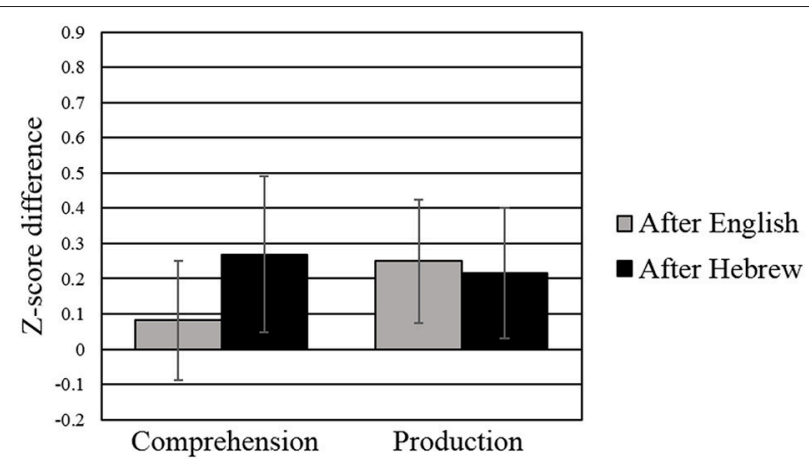

FIGURE 3 | Pre and post z-scores in the category fluency task (error bars represent standard error).

or inhibit their L1 translation equivalents. The fact that the increase in fluency was observed in the letter task, which is less susceptible to the effect of spreading activation between locally related nodes as the semantically-driven category task, further supports a global, rather than item-specific, change in relative activation.

\section{DISCUSSION}

Overall results suggest that even short-term L2 use (less than $10 \mathrm{~min}$ ) significantly increased letter verbal fluency. Furthermore, both comprehension and production of L2 facilitated subsequent fluency. It should be noted that, overall, participants in the English production condition produced shorter and less detailed descriptions than the descriptions presented in the English comprehension condition (trying to limit spoken communication to a minimum, participants were not asked for further clarifications). Accordingly, the lack of mode of use may stem from a disparity in the amount of L2 use and should be interpreted with caution. It should also be noted that mode of use was manipulated only on the interim tasks, the final test involved exclusively a production fluency task. Importantly, an increase in fluency was observed after a purely receptive L2 use, suggesting it does not result from actual output practice. Furthermore it suggests the increase does not result from affective factors such as discomfort and apprehension about speaking out loud in a non-native language that may gradually dissipate as one begins to speak.

The improvement in the letter fluency task further suggest the increase in fluency is due to a global increase in the relative activation of $\mathrm{L} 2$, rather than to the local activation of specific lexical items. Retrieved lexical items were not the same as those encountered during the interim task, and given that the letter task is a phonemic non-semantic task, spreading activation between semantically related lexical nodes cannot account for post-test improvement. These results concur with previous studies suggesting the involvement of global control mechanisms in L2 use. For example, Misra et al. (2012), (see also Guo et al., 2011) found evidence for L1 inhibition in 
a blocked naming task, suggesting a long-lasting inhibition of L1 following L2 use. Van Assche et al. (2013) found that Chinese-English bilinguals had lower phonemic fluency scores in English (their dominant language) after performing the fluency task in Chinese with different phonemic categories, although this was not the case for Dutch-English bilinguals. Van Assche et al. (2013) suggested that due to the larger proportion of cognates for Dutch and English, it may be efficient to keep both languages active. Nevertheless, these results provide evidence for whole-language control mechanisms, at least in some cases. Taken together these findings suggest a global inhibitory mechanism that is both long-lasting and broad. The increase in L2 fluency in the current study was observed across tasks and across different lexical items, and provides further support for a global selection mechanism (at least in late unbalanced bilinguals, for different views regarding the role of proficiency in language selection see Costa (2005) and Kroll et al. (2006).

Finally, improvement was evident in a context that did not involve constant language switching or any uncertainty with respect to the target language (cf. Meuter and Allport, 1999). Fluency tasks were conducted only in English, presented with English written instructions and participants were explicitly told the next tasks will be conducted in English. Moreover, the first session always consisted entirely of English tasks. Thus, resources that participants could exploit in selecting the target language, such as top-down control of the task set and language specific cues (see Kroll et al., 2008), were already available at pretest, as well as to participants in the Hebrew conditions (although, given that the experiment was conducted in a Hebrew speaking environment, with experimenters who were native Hebrew speakers, participants were not in a full English language mode, see Grosjean, 2001). Although the immediate context of the verbal fluency tasks was a single language context in which the target language was fully predictable and explicitly specified, given that our participants were unbalanced bilinguals, with a clear L1 dominance pattern, performing novel L2 tasks that involved non-repeated stimuli, we would expect L1 (i.e., Hebrew) to be active (Kroll et al., 2006). However, actual L2 use may be more effective in bringing about a shift in the relative activation (inhibiting L2 or increasing the activation of L2) than other language selection cues such as task instructions. As a result of the intervening L2 task, L2 was relatively more active at the outset of the fluency posttest, enabling participants to better manage cross-language activation, and freeing up executive resources to deal with the executive demands of the letter fluency task. Interestingly, when vocabulary size is controlled, bilinguals show an advantage in letter fluency, possibly due to a general advantage in cognitive control owing to their experience at negotiating cross-language activation (Bialystok et al., 2008).

These results are consistent with Linck et al. (2009) who found that immersed L2 learners were better at negotiating interference from L1 then classroom L2 learners. Linck et al. further found reduced access to L1 in immersed learners, suggesting inhibition of L1 during immersion. Results suggest that initial adjustment to L2 can temporarily confer some of the benefits provided by longer L2 use, though obviously to a far weaker degree. Furthermore, the current results underscore the importance of recent language experience, even a brief one, in addition to the more stable language dominance pattern. Activation of L1 was not examined in the current study; therefore, our results are also consistent with accounts that suggest that language selection does not involve inhibition of the nontarget language, but rather preferential activation of the target language (Poulisse and Bongaerts, 1994; Grosjean, 2001).

These results are also consistent with Elston-Güttler et al. (2005) who argued that there is a gradual process of adjustment following a switch to L2, a process they coined "zooming into L2." Elston-Güttler et al. examined the processing of German-English homographs embedded in an English context. German speakers who watched a German film prior to the experiment experienced L1 interference during the first half of the experiment, gradually adjusting to L2 language mode. However, German speakers who watched an English film did not experience L1 interference from the outset, suggesting the film served a "zooming in" function. The current research goes beyond these results by showing that L2 use can not only decrease the interference from specific L1 competitors, but globally enhance the accessibility of L2 representations and facilitate subsequent word retrieval.

One question left open concerns the relation between amount of L2 use and the increase in L2 relative activation. Global changes in the relative activation of L2 should increase gradually, rather than operate in an all-or-none manner. For example, in the current study, all participants were presented with English instructions before performing the fluency task, but this was insufficient to bring about a reliable improvement. In addition, with further L2 use, local changes in activation may gradually build-up and propagate through the network. However, it is not clear whether continued L2 use would result in a continuous linear increase in activation. One issue is that with further L2 use, for example in an immersion context, language proficiency is expected to increase. As increased proficiency may be reflected in the level of L2 activation, it may be problematic to disentangle the effects of increased proficiency and increased use on L2 activation. In any case, results show that even a brief period of L2 use, that should not affect proficiency, can benefit L2 fluency. As a practical lesson, if you need to give a talk in English on Monday, better start speaking in English on Sunday.

\section{AUTHOR CONTRIBUTIONS}

HS and MF conceived and designed the experiment, analyzed the results, and wrote the manuscript. HS performed the experiment. 


\section{REFERENCES}

Baldo, J. V., Schwartz, S., Wilkins, D., and Dronkers, N. F. (2006). Role of frontal versus temporal cortex in verbal fluency as revealed by voxelbased lesion symptom mapping. J. Int. Neuropsych. Soc. 12, 896-900. doi: $10.1017 / S 1355617706061078$

Bialystok, E., Craik, F. I. M., Green, D. W., and Gollan, T. H. (2009). Bilingual minds. Psychol. Sci. Public Interest. 10, 89-129. doi: 10.1177/15291006103 87084

Bialystok, E., Craik, F. I. M., and Luk, G. (2008). Lexical access in bilinguals: effects of vocabulary size and executive control. J. Neurolinguist. 21, 522-538. doi: 10.1016/j.jneuroling.2007.07.001

Costa, A. (2005). "Lexical access in bilingual production," in Handbook of Bilingualism: Psycholinguistic Approaches, eds J. F. Kroll and A. M. B. de Groot (Oxford: Oxford University Press), 308-325.

Costa, A., and Santesteban, M. (2004). Lexical access in bilingual speech production: evidence from language switching in highly proficient bilinguals and L2 learners. J. Mem. Lang. 50:491-511. doi: 10.1016/j.jml.2004.02.002

Declerck, M., and Philipp, A. M. (2018). Is inhibition implemented during bilingual production and comprehension? $\mathrm{n}-2$ language repetition costs unchained. Lang. Cogn. Neurosci. 33, 608-617. doi: 10.1080/23273798.2017.1398828

Dijkstra, A. F. J., and Van Heuven, W. J. B. (1998). "The BIA model and bilingual word recognition," in Localist Connectionist Approaches to Human Cognition, eds J. Grainger and A. M. Jacobs (Mahwah, NJ: Erlbaum), 189-225.

Elston-Güttler, K. E., Gunter, T. C., and Kotz, S. A. (2005). Zooming into L2: global language context and adjustment affect processing of interlingual homographs in sentences. Cogn. Brain Res. 25, 57-70. doi: 10.1016/j.cogbrainres.2005. 04.007

Gollan, T. H., Montoya, R. I., and Lerner, G. A. (2002). Semantic and letter fluency in Spanish-English bilinguals. Neuropsychology16, 562-576. doi: 10.1037/0894-4105.16.4.562

Green, D. W. (1998). Mental control of the bilingual lexico-semantic system. Biling. Lang. Cogn. 1, 67-81. doi: 10.1017/S1366728998000133

Grosjean, F. (2001). "The bilingual's language modes," in One Mind, Two Languages: Bilingual Language Processing, ed J. L. Nicol (Cambridge, MA: Blackwell), 1-22.

Guo, T., Liu, H., Misra, M., and Kroll, J. F. (2011). Local and global inhibition in bilingual word production: $\mathrm{fMRI}$ evidence from Chinese-English bilinguals. NeuroImage 56, 2300-2309. doi: 10.1016/j.neuroimage.2011.03.049

Kroll, J. F., Bobb, S. C., Misra, M. M., and Guo, T. (2008). Language selection in bilingual speech: evidence for inhibitory processes. Acta Psychol. 128, 416-430. doi: 10.1016/j.actpsy.2008.02.001

Kroll, J. F., Bobb, S. C., and Wodniecka, Z. (2006). Language selectivity is the exception, not the rule: arguments against a fixed locus of language selection in bilingual speech. Biling. Lang. Cogn. 9, 119-135. doi: $10.1017 / \mathrm{S} 1366728906002483$

Levy, B. J., McVeigh, N. D., Marful, A., and Anderson, M. C. (2007). Inhibiting your native language: the role of retrieval-induced forgetting during second-language acquisition. Psychol. Sci. 18, 29-34. doi: $10.1111 / j .1467-9280.2007 .01844 . x$
Linck, J. A., Kroll, J. F., and Sunderman, G. (2009). Losing access to the native language while immersed in a second language: evidence for the role of inhibition in second language learning. Psychol. Sci. 20, 1507-1515. doi: 10.1111/j.1467-9280.2009.02480.x

Luo, L., Luk, G., and Bialystok, E. (2010). Effect of language proficiency and executive control on verbal fluency performance in bilinguals. Cognition 114, 29-41. doi: 10.1016/j.cognition.2009.08.014

Meuter, R. (2005). "Language selection in bilinguals: mechanisms and processes," in Handbook of Bilingualism: Psycholinguistic Approaches, eds J. F. Kroll and A. M. B. de Groot (Oxford: Oxford University Press), 349-370.

Meuter, R. F. I., and Allport, A. (1999). Bilingual language switching in naming: asymmetrical costs in language selection. J. Mem. Lang. 40, 25-40. doi: 10.1006/jmla.1998.2602

Misra, M., Guo, T., Bobb, S., and Kroll, J. F. (2012). When bilinguals choose a single word to speak: electrophysiological evidence for inhibition of the native language. J. Mem. Lang. 67, 224-237. doi: 10.1016/j.jml.2012.05.001

Portocarrero, J. S., Burright, R. G., and Donovick, P. J. (2007). Vocabulary \& verbal fluency of bilingual and monolingual college students. Arch. Clin. Neuropsych. 22, 415-422. doi: 10.1016/j.acn.2007.01.015

Poulisse, N., and Bongaerts, T. (1994). First language use in second language production. Appl. Linguist. 15, 36-57. doi: 10.1093/applin/15.1.36

Schwartz, S., Baldo, J., Graves, R. E., and Brugger, P. (2003). Pervasive influence of semantics in letter and category fluency: a multidimensional approach. Brain Lang. 87, 400-411. doi: 10.1016/S0093-934X(03)00141-X

Skehan, P. (1996). A framework for the implementation of task-based instruction. Appl. Linguist. 17, 38-62. doi: 10.1093/applin/17.1.38

Tavakoli, P., and Skehan, P. (2005). "Strategic planning, task structure, and performance testing," in Planning and Task Performance in a Second Language, ed R. Ellis (Amsterdam: John Benjamin), 239-277.

Thomas, M. S., and Allport, A. (2000). Language switching costs in bilingual visual word recognition. J. Mem. Lang. 43, 44-66. doi: 10.1006/jmla.1999.2700

Troyer, A. K., Moscovitch, M., and Winocur, G. (1997). Clustering and switching as two components of verbal fluency: evidence from younger and older healthy adults. Neuropsychology 11, 138-146. doi: 10.1037/0894-4105.11.1.138

Van Assche, E., Duyck, W., and Gollan, T. H. (2013). Whole-language and itemspecific control in bilingual language production. J. Exp. Psychol. Learn. Mem. Cogn. 39, 1781-1792. doi: 10.1037/a0032859

$\mathrm{Wu}, \mathrm{Y}$. J., and Thierry, G. (2010). Investigating bilingual processing: the neglected role of language processing contexts. Front. Psychol. 1:178. doi: $10.3389 /$ fpsyg. 2010.00178

Conflict of Interest Statement: The authors declare that the research was conducted in the absence of any commercial or financial relationships that could be construed as a potential conflict of interest.

Copyright (c) 2018 Shintel and Faust. This is an open-access article distributed under the terms of the Creative Commons Attribution License (CC BY). The use, distribution or reproduction in other forums is permitted, provided the original author(s) and the copyright owner(s) are credited and that the original publication in this journal is cited, in accordance with accepted academic practice. No use, distribution or reproduction is permitted which does not comply with these terms. 